\title{
Association Between BRCA1 and BRCA2 Mutations and Survival in Women with Invasive Epithelial Ovarian Cancer
}

Kelly L. Bolton ${ }^{1,2}$, Georgia Chenevix-Trench ${ }^{3}$, Cindy Goh $^{4}$, Siegal Sadetzki ${ }^{5,6}$, Susan J. Ramus $^{7}$, Beth Y. Karlan ${ }^{8}$, Diether Lambrechts ${ }^{9}$, Evelyn Despierre ${ }^{10}$, Daniel Barrowdale ${ }^{11}$, Lesley McGuffog ${ }^{11}$, Sue Healey ${ }^{3}$, Douglas F. Easton ${ }^{11}$, Olga Sinilnikova ${ }^{12,13}$, Javier Benitez $^{14,15}$, María J. García ${ }^{15,16}$, Susan Neuhausen ${ }^{17}$, Mitchell H. Gail ${ }^{1}$, Patricia Hartge ${ }^{1}$, EMBRACE study team ${ }^{11}$, Susan Peock ${ }^{11}$, Debra Frost ${ }^{11}$, D. Gareth Evans ${ }^{18}$, Ros Eeles ${ }^{19}$, Andrew K. Godwin ${ }^{20}$, Mary B. Daly ${ }^{21}$, Ava Kwong ${ }^{22,23}$, Edmond SK Ma ${ }^{22,24}$, Conxi Lázaro ${ }^{25}$, Ignacio Blanco ${ }^{25}$, Marco Montagna ${ }^{26}$, Emma D'Andrea ${ }^{27,28}$, Ornella Nicoletto ${ }^{29}$, kConFab Investigators $^{30}$, Sharon E. Johnatty ${ }^{3}$, Susanne Krüger Kjær ${ }^{31,32}$, Allan Jensen ${ }^{31,32}$, Estrid Høgdall $^{31,32}$, Ellen L. Goode ${ }^{33}$, Brooke L. Fridley ${ }^{33}$, Jennifer T. Loud ${ }^{34}$, Mark H. Greene ${ }^{34}$, Phuong L. Mai ${ }^{34}$, Angela Chetrit ${ }^{5}$, Flora Lubin ${ }^{35}$, Galit Hirsh-Yechezkel ${ }^{5}$, Gord Glendon ${ }^{36}$, Irene L. Andrulis ${ }^{36,37}$, Amanda E. Toland ${ }^{38}$, Leigha Senter ${ }^{39}$, Martin E. Gore ${ }^{40}$, Charlie Gourley $^{41}$, Caroline $\mathbf{O}$ Michie $^{41}$, Honglin Song ${ }^{42}$, Jonathan Tyrer ${ }^{42}$, Alice S. Whittemore ${ }^{43}$, Valerie McGuire ${ }^{43}$, Weiva Sieh ${ }^{43}$, Ulf Kristoffersson ${ }^{44}$, Håkan Olsson ${ }^{45}$, Åke Borg ${ }^{45}$, Douglas A. Levine ${ }^{46}$, Cancer Genome Atlas Research Network ${ }^{47}$, Linda Steele ${ }^{17}$, Mary S. Beattie $^{48,49}$, Salina Chan ${ }^{48}$, Robert Nussbaum ${ }^{48,49}$, Kirsten B. Moysich ${ }^{50}$, Jenny Gross ${ }^{8}$, llana Cass ${ }^{8}$, Christine Walsh ${ }^{8}$, Andrew J. $\mathrm{Li}^{8}$, Ronald Leuchter ${ }^{8}$, Ora Gordon ${ }^{8}$, Montserrat Garcia-Closas $^{51}$, Simon A. Gayther ${ }^{7}$, Stephen J. Chanock ${ }^{1}$, Antonis C. Antoniou ${ }^{11}$, and Paul D.P. Pharoah ${ }^{42}$

${ }^{1}$ Division of Cancer Epidemiology and Genetics, National Cancer Institute, Bethesda MD 20892, USA

2David Geffen School of Medicine at the University of California, Los Angeles CA 90095, USA

${ }^{3}$ Queensland Institute of Medical Research, Locked Bag 2000, Royal Brisbane Hospital, Herston, QLD 4029, Australia

${ }^{4}$ Addenbrooke's Hospital, Hills Road, Cambridge CB2 OQQ, UK

${ }^{5}$ Cancer and Radiation Epidemiology Unit, Gertner Institute for Epidemiology and Health Policy, Sheba Medical Center, Tel Hashomer, 52621, Israel

${ }^{6}$ Sackler Faculty of Medicine, Tel-Aviv University, Tel-Aviv, 69978 Israel

${ }^{7}$ Department of Preventive Medicine, Keck School of Medicine University of Southern California, Los Angeles, California, 90033, USA

${ }^{8}$ Women's Cancer Program at the Samuel Oschin Comprehensive Cancer Institute, Cedars-Sinai Medical Center, Los Angeles, CA, 90048 USA

${ }^{9}$ VIB Vesalius Research Center, University of Leuven, Leuven, 3000 Belgium

${ }^{10}$ Division of Gynaecologic Oncology, Department of Obstetrics and Gynaecology, University Hospitals Leuven, University of Leuven, Leuven, 3000 Belgium

Access to data

Kelly Leigh Bolton had full access to all the data in the study and takes responsibility for the integrity of the data and the accuracy of the data analysis. 
${ }^{11}$ Centre for Cancer Genetic Epidemiology, Department of Public Health and Primary Care, University of Cambridge, Strangeways Research Laboratory, Worts Causeway, Cambridge CB1 8RN, UK

${ }^{12}$ Unité Mixte de Génétique Constitutionnelle des Cancers Fréquents, Centre Hospitalier Universitaire de Lyon, Lyon 69373, France

${ }^{13}$ Centre Léon Bérard, and Equipe labellisée LIGUE 2008, UMR5201 CNRS, Centre Léon Bérard, Université de Lyon, Lyon 69373, France

${ }^{14}$ Human Cancer Genetics Programme and Genotyping Unit, Spanish National Cancer Research Centre, Melchor Fernández Almagro 3, Madrid 28029, Spain

${ }^{15}$ CIBERER, Melchor Fernández Almagro 3, Madrid 28029, Spain

${ }^{16}$ Human Genetics Group, Human Cancer Genetics Programme, Spanish National Cancer Research Centre, Melchor Fernández Almagro 3, Madrid 28029, Spain

${ }^{17}$ Department of Population Sciences, the Beckman Research Institute of the City of Hope, Duarte, CA 91010, USA

${ }^{18}$ Genetic Medicine, Manchester Academic Health Sciences Centre, Central Manchester University Hospitals NHS Foundation Trust, Manchester, M13 9WL UK

${ }^{19}$ Oncogenetics Team, The Institute of Cancer Research and Royal Marsden NHS Foundation Trust, SM2 5NG UK

20Department of Pathology and Laboratory Medicine, The University of Kansas Medical Center, Kansas City, KS 66160, USA

${ }^{21}$ Department of Clinical Genetics, Fox Chase Cancer Center, Philadelphia, PA 19111, USA

${ }^{22}$ The Hong Kong Hereditary Breast Cancer Family Registry, Cancer Genetics Centre, Hong Kong Sanatorium and Hospital, 4/F, Central Block, 2 Village Road, Hong Kong

${ }^{23}$ Division of Breast Surgery, The University of Hong Kong, Queen Mary Hospital, Hong Kong

${ }^{24}$ Division of Molecular Pathology, Hong Kong Sanatorium and Hospital, 4/F, Central Block, 2 Village Road, Hong Kong

${ }^{25}$ Hereditary Cancer Programme, Catalan Institute of Oncology, IDIBELL, Gran Via 199-203, L'Hospitalet, Barcelona 08907, Spain

${ }^{26}$ Immunology and Molecular Oncology Unit, Istituto Oncologico Veneto - IRCCS, Via Gattamelata 64, 35128 Padua, Italy

${ }^{27}$ Department of Oncology and Surgical Sciences, University of Padua, 35128 Padua, Italy

${ }^{28}$ Istituto Oncologico Veneto IRCCS, Via Gattamelata 64, 35128 Padua, Italy

${ }^{29}$ Medical Oncology Unit, Istituto Oncologico Veneto - IRCCS, Via Gattamelata 64, 35128 Padua, Italy

30Peter MacCallum Cancer Centre, East Melbourne, VIC 3002, Australia

${ }^{31}$ Department of Virus, Hormones and Cancer, Danish Cancer Society, Copenhagen, 2100 Denmark

${ }^{32}$ Department of Gynecology, Rigshospitalet, University of Copenhagen, Copenhagen, 2100 Denmark

${ }^{33}$ Department of Health Sciences Research, Mayo Clinic College of Medicine, Rochester, Minnesota 55905, USA 
${ }^{34}$ Clinical Genetics Branch, Division of Cancer Epidemiology and Genetics, National Cancer Institute, Rockville MD 20852, USA

${ }^{35}$ Gertner Institute for Epidemiology and Health Policy, Sheba Medical Center, Tel Hashomer, 52621 , Israel

${ }^{36}$ Ontario Cancer Genetics Network, Cancer Care Ontario, Toronto, Ontario, M5G 2C1 Canada

${ }^{37}$ Samuel Lunenfeld Research Institute, Mount Sinai Hospital, Toronto, Ontario, M5G 1X5 Canada

${ }^{38}$ Division of Human Cancer Genetics, Departments of Internal Medicine and Molecular Virology, Immunology and Medical Genetics, The Comprehensive Cancer Center, The Ohio State University, Columbus, USA

${ }^{39}$ Clinical Cancer Genetics Program, Division of Human Genetics, Department of Internal Medicine, The Comprehensive Cancer Center, The Ohio State University, Columbus, 43210 USA ${ }^{40}$ Gynecological Oncology Unit, The Royal Marsden Hospital, FulhamRoad, London SW3 6JJ, UK

${ }^{41}$ University of Edinburgh Cancer Research Centre, Institute of Genetics and Molecular Medicine, Western General Hospital, Edinburgh, EH4 2XR UK

${ }^{42}$ Cancer Research United Kingdom, Department of Oncology and Department of Public Health and Primary Care, University of Cambridge, Strangeways Research Laboratory, Worts Causeway, Cambridge CB1 8RN, UK

${ }^{43}$ Department of Health Research and Policy, Stanford University School of Medicine, Stanford, CA 94305, USA

${ }^{44}$ Department of Clinical Genetics, University and Regional Laboratories Skåne and Lund University, SE-20502 Lund, Sweden

${ }^{45}$ Department of Oncology, Lund University, SE-22185 Lund, Sweden

${ }^{46}$ Gynecology Service, Department of Surgery, Memorial Sloan-Kettering Cancer Center, New York, NY, 10065 USA

${ }^{47}$ National Cancer Institute at National Institutes of Health, Bethesda, MD 20892, USA

${ }^{48}$ UCSF Cancer Risk Program, San Fransisco, CA 94143, USA

${ }^{49}$ UCSF Departments of Medicine, San Fransisco, CA 94118, USA

${ }^{50}$ Department of Cancer Prevention and Control, Roswell Park Cancer Institute, Buffalo, NY 14263, USA

${ }^{51}$ Sections of Epidemiology and Genetics, Institute of Cancer Research and Breakthrough Breast Cancer Research Centre, London, SW7 3RP UK

\section{Abstract}

Context-Approximately 10 percent of women with invasive epithelial ovarian cancer (EOC) carry deleterious germline mutations in $B R C A 1$ or $B R C A 2$. A recent report suggested that $B R C A 2$ related EOC was associated with an improved prognosis, but the effect of BRCA1 remains unclear.

Objective-To characterize the survival of $B R C A$ carriers with EOC compared to non-carriers and to determine whether $B R C A 1$ and $B R C A 2$ carriers show similar survival patterns.

Design, Setting, and Participants-We pooled data from 26 studies on the survival of women with ovarian cancer. This included data on 1,213 EOC cases with pathogenic germline 
mutations in BRCA1 (909) or BRCA2 (304) and 2,666 non-carriers recruited and followed for variable times between 1987 and 2010; the median year of diagnosis was 1998.

Main Outcome Measures-Five year overall mortality.

Results-The five-year overall survival was 36 percent (95\% CI: 34-38) for non-carriers, 44 percent (95\% CI: 40-48) for BRCA1 carriers and 52 percent (95\% CI: 46-58) for BRCA2 carriers. After adjusting for study and year of diagnosis, $B R C A 1$ and $B R C A 2$ carriers showed a more favorable survival than non-carriers $\left(B R C A 1, \mathrm{HR}=0.78 ; 95 \% \mathrm{CI}=0.68-0.89, \mathrm{P}=2 \times 10^{-4}\right.$; $\left.B R C A 2, \mathrm{HR}=0.61 ; 95 \% \mathrm{CI}=0.50-0.76, \mathrm{P}=6 \times 10^{-6}\right)$. These survival differences remained after additional adjustment for stage, grade, histology and age at diagnosis $(B R C A 1, \mathrm{HR}=0.73,95 \%$ $\left.\mathrm{CI}=0.64-0.84, \mathrm{P}=2 \times 10^{-5} ; B R C A 2, \mathrm{HR}=0.49,95 \% \mathrm{CI}=0.39-0.61, \mathrm{P}=3 \times 10^{-10}\right)$.

Conclusions-Among patients with invasive epithelial ovarian cancer, having a germline mutation in $B R C A 1$ or $B R C A 2$ was associated with improved 5-year overall survival.

\section{Introduction}

Germline mutations in the genes $B R C A 1$ and $B R C A 2$ are the strongest known genetic risk factors for both breast and epithelial ovarian cancer (EOC) and are found in 6-15 percent of women with $\mathrm{EOC}^{1-3}$. BRCA1 is involved in DNA repair, cell-cycle checkpoint control, chromatin remodeling, transcriptional regulation and mitosis and BRCA2 has an important role in homologous recombination ${ }^{4}$. The clinical characteristics of EOCs among BRCA1/2 carriers differ from that of non-carriers. $B R C A 1$ related disease is more likely to be of serous histology ${ }^{5}$, high grade ${ }^{6}$ and advanced stage ${ }^{3}$. Less data are available for $B R C A 2-$ related EOC due to their lower prevalence and lower EOC penetrance relative to BRCA1 but a similar pattern is generally reported $\mathrm{d}^{5 ; 7}$.

The relative prognosis of $B R C A 1 / 2$ carriers and non-carriers is unclear. A recent report found a more favorable outcome for $B R C A 2$ mutation carriers, with no significant difference in outcome for $B R C A 1$ mutation carriers compared to non-carriers ${ }^{8}$. However, some studies have demonstrated a more favorable prognosis for BRCA1 and BRCA2 carriers $6 ; 7 ; 9$ compared to non-carriers whereas others have reported no significant difference ${ }^{10 ; 11}$. Several factors may account for these divergent results. Most studies contained fewer than 50 carriers and all contained fewer than 250 carriers resulting in imprecise survival estimates. Small sample sizes have also resulted in the grouping of $B R C A 1$ and $B R C A 2$ carriers together for analysis, despite potential prognostic differences. In addition, adjustment for prognostic factors known to differ by carrier status has varied among studies. Finally, few studies employed appropriate statistical methods to account for the potential bias that results from the inclusion of prevalent cases ${ }^{12}$. The mechanism driving the association between BRCA1/2 mutations and survival is not known but some retrospective studies suggested that the survival advantage of carriers could be mediated through improved response to platinum-based agents $7 ; 13$. This is consistent with in vitro studies showing that $B R C A 1$ and $B R C A 2$ deficient cells are hypersensitive to drugs which induce double strand DNA breaks such as platinum-based agents ${ }^{14}$.

The aim of this study was to collate the data from multiple EOC case series with data on $B R C A 1$ and $B R C A 2$ mutation status in order to provide definitive evidence of the relative effect of germline $B R C A 1$ and $B R C A 2$ mutations on prognosis. The results could provide insight into the biology of $B R C A 1 / 2$ mutations, improve clinical management of mutation carriers and have implications for clinical trial design, particularly for agents targeting BRCA1/2 dysfunction such as poly (ADP-ribose)-polymerase (PARP) inhibitors ${ }^{15}$. 


\section{Methods}

\section{Study Design}

Study participants were women with confirmed invasive EOC both with and without pathogenic mutations in BRCA1 and BRCA2. Participants were drawn from 26 studies: 10 from the USA, six from Europe, two from Israel, one from Hong Kong, one from Canada, one from Australia and five from the UK. Participants were enrolled in clinical research protocols between 1987 and 2010 that were approved by local institutional review boards. Written consent was obtained from all living patients. Most participating studies were affiliated with either the Consortium of Investigators of Modifiers of BRCA1/2 (CIMBA) ${ }^{16}$ or the Ovarian Cancer Association Consortium (OCAC) ${ }^{17}$. Investigators submitted data on patient demographics, tumor pathology, vital status and treatment to the coordinating group in Cambridge. In some studies, EOC cases were recruited based on a strong family history of ovarian and/or breast cancer (family-based), while others used population-based sampling or enrolled a consecutive series of cases treated at a single or multiple institution(s). In all studies, BRCA1/2 carriers and non-carriers were enrolled into the study using the same criteria.

Mutations were considered pathogenic if they met criteria defined by the Breast Cancer Information Core ${ }^{18 ; 19}$ and were grouped into categories based on their predicted functional effect $^{20-23}$. Women with variants of unknown significance in $B R C A 1$ or $B R C A 2$ were excluded. Class I mutations are the most frequent and represent loss-of-function mutations predicted to result in reduced transcript or protein level due to mRNA nonsense-mediated RNA decay, translational retention or absence of expression. Class II contains those mutations likely to generate stable proteins that may have some normal or dominant negative function. This includes missense substitutions and mutations generating a premature stop codon in the last exon. All participants were screened for both BRCA1 and $B R C A 2$ mutations with three exceptions. In three family-based studies, the Kathleen Cuningham Consortium for Research into Familial Breast Cancer, the UK Gilda Radner Familial Ovarian Cancer Registries and the National Cancer Institute study, some EOC cases were not tested for BRCA1/2 and BRCA1/2 status was assumed to be same as that of affected family member(s) who had been tested. The non-carrier group from the RMH study contained some untested EOC cases but who reported no family history of breast or ovarian cancer and were therefore considered unlikely to harbor mutations. Finally, in the Stanford Genetic Epidemiology of Ovarian Cancer study, only BRCA1 mutation testing was performed. A variety of methods were used to perform mutation testing (eTable 1).

Data on tumor pathology, vital status and treatment were obtained through a combination of medical records, local cancer registries and death certificates. Infrequently, vital status was determined through direct contact with a physician or family member of the patient. In a subset of studies, information regarding residual disease following primary surgery was available from medical records. Optimal debulking was defined as residual disease $=<1 \mathrm{~cm}$ and suboptimal debulking as residual disease $>1 \mathrm{~cm}$.

$B R C A 1 / 2$ status may modify response to platinum based chemotherapy which became standard of care in most countries around 1990. Among the 36 percent of subjects with chemotherapy data, 95 percent of cases diagnosed after 1990 were reported to have received a platinum-based agent. We therefore excluded women diagnosed before 1990 if chemotherapy regime was unknown, and those known not to have received platinum based chemotherapy. 


\section{Statistical Analysis}

The primary endpoint was overall survival (OS) up to five years following EOC diagnosis. We chose this endpoint in order to minimize the influence of non-EOC related deaths. Timeto-event (death or censoring) was calculated from the date of diagnosis. However, cases were recruited at variable times after diagnosis and so time under observation was calculated from date of recruitment (left truncation) in order to prevent the bias that could result from the inclusion of prevalent cases. Effect estimates from left-truncated data are considered to be unbiased if the event time and delayed entry time are independent, given the covariates ${ }^{24}$. Differences in tumor stage, grade, histology and age at diagnosis between BRCA1, BRCA2 and non-carriers were tested using logistic regression adjusted for study site. We used Cox proportional-hazards models to estimate hazard ratios (HR) and 95 percent confidence intervals (CI). All models were adjusted for year of EOC diagnosis (<1990, 1990-1995, 1996-2000, 2000-2010) and stratified by study site. In stratified survival analyses, strata with small numbers of deaths can lead to unreliable estimates. For this reason, four studies with less than 30 cases were placed in the same strata as other studies sharing similar study designs and baseline survival rates.

We performed analyses with and without adjustment for stage, grade, histology and age at diagnosis. The proportional hazards assumption was tested for each covariate analytically using Schoenfeld residuals. Age at diagnosis and histology violated the PH assumption so additional covariates were included to allow for time-dependent effects

Differences in the HR estimates for the survival impact of $B R C A 1$ and $B R C A 2$ by different clinical factors were tested using Cochrane's chi-square test (Q-test) for heterogeneity. To assess the impact of possible competing mortality from breast cancer on effect estimates, we compared analyses restricted to women with and without a diagnosis of breast cancer before or in the five years following EOC diagnosis. We tested for heterogeneity by study in the HR estimates through the inclusion of an interaction term between study and BRCA1/2 mutation status.

Some participants were missing data for stage (19\%), grade (22\%) and histology (5\%). In order to decrease potential bias and loss of power due to missingness, we performed multiple imputation for these three variables (eMethods). All analyses, except for comparison of pathological characteristics and Kaplan Meier estimation of survival, were performed on the imputed data. The results using non-imputed data were similar to those presented here using imputed data; for comparison, the main results using non-imputed data are presented in eTable 2. All analyses were performed using STATA/SE version 11 (StataCorp, College Station, TX, USA). Statistical significance was defined as a P value of less than 0.05. Statistical tests were two sided.

\section{Results}

Data were available for 3,879 EOC cases; 909 BRCA1 and 304 BRCA2 mutation carriers and 2,666 non-carriers. The median number of months from ascertainment to diagnosis for participants was 1 month (25th-75th percentile: $0-15$ months). Women were under active follow-up for a median time of 38 months (25th-75th percentile: $18-77$ months). The proportion of cases with censored survival time (not followed to death or 5 years after diagnosis) was 15 percent. After controlling for study site, there was no significant difference in the proportion of cases with censored survival time among $B R C A 1(\mathrm{p}=0.22)$ or $B R C A 2(\mathrm{p}=0.41)$ carriers compared to non-carriers. The median year of diagnosis was 1998 (range: 1981-2010). During the five years following EOC diagnosis, 1,766 deaths occurred. We found several significant differences in the clinical features of BRCA1 and BRCA2 carriers compared to non-carriers (Table 1). Tumors in $B R C A 1$ and $B R C A 2$ carriers were 
more likely to be of serous histology and less likely to be of mucinous histology than tumors in non-carriers. $B R C A 1$ and $B R C A 2$ carriers were more likely to have stage III/IV tumors and poorly differentiated/undifferentiated tumors than non-carriers. Compared to BRCA1 carriers, $B R C A 2$ carriers were more likely to have stage III/IV tumors. While BRCA1 carriers were younger at diagnosis than non-carriers, $B R C A 2$ carriers were slightly older.

The five-year overall survival was 36 percent (95\% CI: 34-38) for non-carriers, 44 percent (95\% CI: 40-48) for BRCA1 carriers and 52 percent (95\% CI: 46-58) for BRCA2 carriers (Figure 1 and eFigure 1). In a Cox regression model only adjusted for study and year of diagnosis, $B R C A 1$ carriers showed a more favorable survival than non-carriers $(\mathrm{HR}=0.78$; 95\% $\mathrm{CI}=0.68-0.89 ; \mathrm{P}=2 \times 10^{-4}$ ) (Table 2 ). This improved slightly after additional adjustment for stage, grade, histology and age at diagnosis $\left(\mathrm{HR}=0.73 ; 95 \% \mathrm{CI}=0.64-0.84 ; \mathrm{P}=2 \times 10^{-5}\right)$. $B R C A 2$ carriers showed a greater survival advantage compared to non-carriers $(\mathrm{HR}=0.61$; $95 \% \mathrm{CI}=0.50-0.76, \mathrm{P}=6 \times 10^{-6}$ ), particularly after adjusting for other prognostic factors (HR $\left.=0.49 ; 95 \% \mathrm{CI}=0.39-0.61, \mathrm{P}=3 \times 10^{-10}\right)$. The $B R C A 1 \mathrm{HR}$ estimates were significantly different from the $B R C A 2 \mathrm{HR}$ estimates in unadjusted $\left(\mathrm{P}_{\text {het }}=0.05\right)$ and adjusted models $\left(\mathrm{P}_{\text {het }}=0.003\right)$.

We studied the impact of $B R C A 1 / 2$ mutation status on all-cause mortality after stratifying patients by other clinical features (Table 3 ). In analyses stratified by grade and adjusted for other prognostic factors the HRs were $>1$ for both $B R C A 1$ vs. non-carriers and $B R C A 2$ vs. non-carriers in low grade cases but $<1$ in high grade cases. There were no significant differences in the HRs for $B R C A 1$ vs. non-carriers or $B R C A 2$ vs. non-carriers when stratified according to tumor stage, histology or history of breast cancer before or during the study period. The survival advantage of $B R C A 1$ and $B R C A 2$ carriers compared to noncarriers was found to be attenuated in women with ovarian cancer selected based on family history of ovarian and/or breast cancer (Table 4). However, the difference in survival between BRCA1 and BRCA2 carriers did not depend on ascertainment (HR for BRCA2 vs. BRCA1: $0.71,95 \% \mathrm{CI}=0.52-0.98$ and $0.64,95 \% \mathrm{CI}=0.45-0.91$ for familial and unselected cases respectively; $\left.\mathrm{P}_{\text {het }}=0.65\right)$. There was no evidence of study-specific heterogeneity in the HR estimates for mutation status among family-based studies (BRCA1, $\mathrm{p}=0.22 ; B R C A 2$, $\mathrm{p}=0.92)$ or unselected studies (BRCA1, $\mathrm{p}=0.73 ; B R C A 2, \mathrm{p}=0.57)$.

The proportion of mutation carriers with the Ashkenazi Jewish founder mutations 185delAG and 5382insC in BRCA1 and 6174delT in BRCA2 was 26 percent. We did not find any significant differences in the adjusted HRs for $B R C A 1$ vs. non-carriers among carriers by mutation type (Class I vs. Class II mutation $\mathrm{P}_{\text {het }}=0.10$ ). However, the survival advantage of BRCA1 mutation carriers with Class I mutations differed depending on mutation location; worse survival was associated with mutations on the $5^{\prime}$ end compared to the $3^{\prime}$ end of BRCA1 ( $\mathrm{P}=0.03)$ (eMethods and eTable 3).

A subset of 1129 patients had information on residual disease following primary surgery. We assessed the impact of lack of adjustment for these variables in our main analysis by comparing results with and without adjustment for residual disease in this subgroup. Optimal debulking occurred in $85 \%$ of non-carriers, $87 \%$ of BRCA 1 carriers and $91 \%$ of $B R C A 2$ carriers. After adjusting for study site and year of diagnosis, there was no significant difference in the likelihood of optimal debulking between non-carriers and $B R C A 1(\mathrm{p}=0.74)$ or $B R C A 2$ ( $\mathrm{p}=0.46)$ carriers. Adjustment for residual disease did not substantially change the HR estimates for the relative survival of either BRCA1 or BRCA2 carriers compared to non-carriers (eTable 4). 


\section{Discussion}

Our data demonstrate an improved survival in EOC patients with germline BRCA1 and $B R C A 2$ mutations relative to non-carriers, with BRCA2 carriers having the best prognosis. $B R C A 1$ carriers presented with EOC at an earlier age than $B R C A 2$ carriers which is consistent with the age-specific penetrances for $B R C A 1$ compared to $B R C A 2$ carriers. The pathological characteristics of $B R C A 1$ and $B R C A 2$ related tumors are similar to each other, but differ from those of tumors in non-carriers. This contrasts with breast cancer, in which substantial differences between BRCA1 and BRCA2-associated disease are present ${ }^{25 ; 26}$. The differences in grade, stage and histology by mutation status are consistent with previously reported data ${ }^{5 ; 27}$. The impact of $B R C A 1$ and $B R C A 2$ mutations on survival appeared to be similar among patients with both localized and advanced stage tumors and among both serous and non-serous tumors. The lack of a survival advantage for BRCA1 and $B R C A 2$ mutation carriers with low grade disease suggests that disruptions of the BRCA1/2 pathways may not be as important in the etiology of these tumors, supporting evidence of etiologic heterogeneity between high grade and low grade serous carcinoma from other studies ${ }^{28 ; 29}$. However, these results were based on small numbers and require confirmation in larger studies.

Our findings confirm the findings of recent analysis of data from the Cancer Genome Atlas (TCGA) project which reported an improved prognosis for $B R C A 2$ carriers ${ }^{8}$. In contrast we also found an improved prognosis for $B R C A 1$ carriers, whereas the TCGA data suggested no difference between $B R C A 1$ carriers and non-carriers. The most likely reason for this difference is the lack of power to detect a moderate difference in survival in the TCGA data. Indeed, the hazard ratio for BRCA1 carriers compared to non-carriers reported by Yang and colleagues (multivariate adjusted $\mathrm{HR}=0.76$ ) was very similar to that from our analysis (multivariate adjusted $\mathrm{HR}=0.73$ ).

We found a smaller survival effect of $B R C A 1$ and $B R C A 2$ in the subset of studies where participants selected based on a strong family history of ovarian and/or breast cancer. This could have been due to misclassification of non-carriers in these studies. The sensitivity of mutation testing is likely to be similar across all studies but the proportion of false negative carriers will be higher in familial cases. Alternatively, cases from BRCA1/2 wild-type families could carry germline mutations in genes in the same pathway as BRCA1/2 (such as RAD $51 C^{30}$ ) or in different pathways that produce similar clinical features.

The improved survival of $B R C A 1 / 2$ carriers relative to non-carriers, and the survival advantage of $B R C A 2$ carriers relative to $B R C A 1$ carriers could be related to intrinsic biological differences, their response to therapeutic agents or both. In addition to differences in stage, grade and histology, BRCA1/2 carriers could have differences in other aspects of tumor biology that were not measured in the current study. For example, BRCA1 and $B R C A 2$ carriers have been recently shown to differ from each other and from sporadic EOC in the incidence of visceral metastasis ${ }^{31}$.

The most notable advantage as well as disadvantage of our study is the fact that it is based on a heterogeneous population; these data were taken from studies containing different ethnic groups, employing different mutation screening methodologies and case ascertainment. By including a wide variety of studies, we were able to generate a large enough sample size to adequately address the issue of heterogeneity of the survival effect between $B R C A 1$ and $B R C A 2$ carriers. But, differences in study design and population may limit the specificity of the conclusions drawn. Additionally, varying levels of misclassification of $B R C A$ status and other variables of interest may have led to some bias of our estimates towards the null. However, the absence of heterogeneity in study-specific 
effects (after accounting for selection on family history) suggests that these results are generalizable to many populations. Furthermore, the magnitude of the differences we observed between $B R C A 1, B R C A 2$ carriers and non-carriers, despite the presence of heterogeneity, provide further testament to their robustness. Even at the lower bounds of our effect estimates, $B R C A 2$ carriers would be predicted to show a $64 \%$ decreased risk of death in the five years following diagnosis compared to non- carriers.

Our findings could have relevance to an even higher proportion of EOC patients if somatic mutations and epigenetic silencing of BRCA1 and BRCA2 show similar effects on prognosis to germline mutations. It has been estimated that roughly $30 \%$ of $\mathrm{EOC}$ and over half of high-grade serous EOC could show dysfunction of $B R C A 1$ or $B R C A 2$ through genetic or epigenetic events ${ }^{32 ; 33}$. There is evidence that EOC cases with somatic BRCA1/2 mutations show a survival advantage over non-carriers ${ }^{33}$, but data from The Cancer Genome Atlas and others suggest that silencing of BRCA1 through promoter methylation does not result in an improved $\mathrm{OS}^{34 ; 35}$. Larger studies that include comprehensive genomic screening of $B R C A 1$ and $B R C A 2$ in primary EOCs will be needed determine if alterations at the somatic and epigenetic level have similar clinical effects to germline mutations.

The results of this study have potentially important implications for the clinical management of patients with EOC. Most immediately, our findings can be used by health care professions for patient counseling regarding expected survival. BRCA1 and BRCA2 carriers with EOC respond better than non carriers to platinum based chemotherapies, and have improved survival despite the fact that the disease is generally diagnosed at a later stage and higher grade. If patients could be stratified based on their BRCA status, their treatment could be tailored to reflect this, with non-carriers targeted for more aggressive treatments. Our data provide further support that there may be different functional mechanisms involved in the etiology of different subtypes of EOCs, and therefore different therapeutic targets based on germline and somatic genetic variation. For example, the functional characterization of BRCA1 and BRCA2 led to the development of a novel therapy in $B R C A 1 / 2$ carriers based on inhibition of the poly (ADP-ribose) polymerase (PARP) DNA repair pathway, creating a synthetic lethal phenotype. Recently, phase I and II trials have shown anti-tumor activity of the PARP inhibitor Olparib in BRCA1/2 mutation carriers with $\mathrm{EOC}^{15 ; 36 ; 37}$. These trials were not large enough to detect differences in response to Olparib in $B R C A 1$ vs. $B R C A 2$ carriers and it is not known whether they will show similar levels of response. EOC clinical trials should be stratified by $B R C A$ status not only to more appropriately target therapy but also to avoid the potential bias introduced by unequal numbers of carriers in treatment arms or between study cohorts. Furthermore, given the important prognostic information provided by $B R C A 1$ and $B R C A 2$ status and the potential for personalized treatment in carriers, the routine testing of women presenting with highgrade serous EOC may now be warranted.

\section{Supplementary Material}

Refer to Web version on PubMed Central for supplementary material.

\section{Acknowledgments}

We thank all the patients and families who took part in the component studies and the many individuals who have made these studies possible. In particular we thank John Stratton, $\mathrm{PhD}$ and Vickie Basham, BA for their help in collecting and managing data from the UK Gilda Radner Familial Ovarian Cancer Registry study. We thank Heather Thorne, BSci, Professional Diploma in Clinical Research and Eveline Niedermayr, B.Sc, G.Dip. Information Management, G.Dip. Software Development for their help in collecting and managing data for the Kathleen Cuningham Consortium for Research into Familial Breast Cancer study. None of the individuals acknowledged here received specific compensation for their role in this article. 
The contributing studies are supported by Cancer Research UK (C490/A10119, C490/A10124, C1287/A10118, C1287/A11990, C5047/A8385), the Edinburgh Experimental Cancer Medicine Centre, the European Community's Seventh Framework Programme ( ${ }^{\circ}$ 223175, HEALTH-F2-2009-223175), the Fondo de Investigación Sanitaria (PS09/01094 \& PI081120), the Fundación Mutua Madrileña (AP-8101-2010), the Melville Trust for the Care and Cure of Cancer, the National Institutes of Health (CA74415, R01-CA 61107, R01 CA-122443, P50 CA136393, P01 CA 130818, 2P50 CA 058207 and the Intramural Research Program of the NCI, DCEG), the Nationaal Kankerplan - Actie 29' of Belgium, the NHMRC of Australia, the National Breast Cancer Foundation of Australia, Cancer Australia (\#628333), the Queensland Cancer Fund, the Cancer Councils of New South Wales, Victoria, Tasmania and South Australia, the Cancer Foundation of Western Australia, the Scottish Funding Council, the Scottish Chief Scientist's Office, the Manchester NIHR Biomedical Research Centre, Minnesota Ovarian Cancer Alliance, Fred C. and Katherine B. Andersen Foundation, Mermaid 1 project, the Danish Cancer Society, Ministero dell'Istruzione, dell'Università e della Ricerca and Ministero della Salute ("Progetto Tumori Femminili" and RFPS 2006-5-341353, ACC2/R6.9"), the Helen Diller Family Comprehensive Cancer Center at UCSF, the Avon Foundation, The Hong Kong Hereditary Breast Cancer Family Registry and the Dr. Ellen Li Charitable Foundation and Service Grants through the National Cancer Institute (NO2-CP-11019-50 and N02-CP-65504 with Westat, Inc, Rockville, MD), Asociación Española Contra el Cáncer, Spanish Health Research Fund; Carlos III Health Institute; Catalan Health Institute and Autonomous Government of Catalonia, Contract grant numbers (ISCIIIRETIC RD06/0020/1051, PI10/01422, PI10/31488 and 2009SGR290). B.Y.K is supported by funding from the American Cancer Society Clinical Research Professorship (\#SIOP-06-258-06-COUN). M.J.G. is the recipient of a Miguel Servet contract from the Instituto de Salud Carlos III, A.C.A is a Cancer Research UK Senior Cancer Research Fellow.

\section{Role of Sponsors}

None of the sponsors described above had any role in the design or conduct of the study; the collection, analysis, and interpretation of the data; or the preparation, review, or approval of the manuscript.

\section{Reference List}

1. Zhang S, Royer R, Li S, et al. Frequencies of BRCA1 and BRCA2 mutations among 1,342 unselected patients with invasive ovarian cancer. Gynecologic Oncology. 2011; 121:353-357. [PubMed: 21324516]

2. Pal T, Permuth-Wey J, Betts JA, et al. BRCA1 and BRCA2 mutations account for a large proportion of ovarian carcinoma cases. Cancer. 2005; 104:2807-2816. [PubMed: 16284991]

3. Soegaard M, Kjaer SK, Cox M, et al. BRCA1 and BRCA2 Mutation Prevalence and Clinical Characteristics of a Population-Based Series of Ovarian Cancer Cases from Denmark. Clin Cancer Res. 2008; 14:3761-3767. [PubMed: 18559594]

4. O'Donovan PJ, Livingston DM. BRCA1 and BRCA2: breast/ovarian cancer susceptibility gene products and participants in DNA double-strand break repair. Carcinogenesis. 2010; 31:961-967. [PubMed: 20400477]

5. Lakhani SR, Manek S, Penault-Llorca F, et al. Pathology of ovarian cancers in BRCA1 and BRCA2 carriers. Clin Cancer Res. 2004; 10:2473-2481. [PubMed: 15073127]

6. Chetrit A, Hirsh-Yechezkel G, Ben-David Y, Lubin F, Friedman E, Sadetzki S. Effect of BRCA1/2 mutations on long-term survival of patients with invasive ovarian cancer: the national Israeli study of ovarian cancer. J Clin Oncol. 2008; 26:20-25. [PubMed: 18165636]

7. Cass I, Baldwin RL, Varkey T, Moslehi R, Narod SA, Karlan BY. Improved survival in women with BRCA-associated ovarian carcinoma. Cancer. 2003; 97:2187-2195. [PubMed: 12712470]

8. Yang D, Khan S, Sun Y, et al. Association of BRCA1 and BRCA2 mutations with survival, chemotherapy sensitivity, and gene mutator phenotype in patients with ovarian cancer. JAMA. 2011; 306:1557-1565. [PubMed: 21990299]

9. Boyd J. Clinicopathologic features of BRCA-linked and sporadic ovarian cancer. J Am Med Assoc. 2000; 283:2260-2265.

10. Buller RE, Shahin MS, Geisler JP, Zogg M, De Young BR, Davis CS. Failure of BRCA1 Dysfunction to Alter Ovarian Cancer Survival. Clin Cancer Res. 2002; 8:1196-1202. [PubMed: 12006538]

11. Pharoah PD, Easton DF, Stockton DL, Gayther S, Ponder BA. Survival in familial, BRCA1associated, and BRCA2-associated epithelial ovarian cancer. United Kingdom Coordinating Committee for Cancer Research (UKCCCR) Familial Ovarian Cancer Study Group. Cancer Res. 1999; 59:868-871. [PubMed: 10029077] 
12. Brookmeyer, R. Biased Sampling of Cohorts. In: Armitage, P.; Colton, T., editors. Encyclopedia of Biostatistics. 2. John Wiley \& Sons, Ltd; 2005.

13. Tan DS, Rothermundt C, Thomas K, et al. "BRCAness" syndrome in ovarian cancer: a casecontrol study describing the clinical features and outcome of patients with epithelial ovarian cancer associated with BRCA1 and BRCA2 mutations. J Clin Oncol. 2008; 26:5530-5536. [PubMed: 18955455]

14. Foulkes WD. BRCA1 and BRCA2: chemosensitivity, treatment outcomes and prognosis. Fam Cancer. 2006; 5:135-142. [PubMed: 16736282]

15. Audeh MW, Carmichael J, Penson RT, et al. Oral poly(ADP-ribose) polymerase inhibitor olaparib in patients with BRCA1 or BRCA2 mutations and recurrent ovarian cancer: a proof-of-concept trial. Lancet. 2010; 376:245-251. [PubMed: 20609468]

16. Chenevix-Trench G, Milne R, Antoniou A, et al. An international initiative to identify genetic modifiers of cancer risk in BRCA1 and BRCA2 mutation carriers: the Consortium of Investigators of Modifiers of BRCA1 and BRCA2 (CIMBA). Breast Cancer Research. 2007; 9:104. [PubMed: 17466083]

17. Pearce CL, Wu AH, Gayther SA, et al. Progesterone receptor variation and risk of ovarian cancer is limited to the invasive endometrioid subtype: results from the Ovarian Cancer Association Consortium pooled analysis. Br J Cancer. 2008; 98:282-288. [PubMed: 18219286]

18. Szabo C, Masiello A, Ryan JF, Brody LC. The Breast Cancer Information Core: Database design, structure, and scope. Human Mutation. 2000; 16:123-131. [PubMed: 10923033]

19. Antoniou AC, Sinilnikova OM, Simard J, et al. RAD51 135G-->C Modifies Breast Cancer Risk among BRCA2 Mutation Carriers: Results from a Combined Analysis of 19 Studies. The American Journal of Human Genetics. 2007; 81:1186-1200.

20. Buisson M, Anczukow O, Zetoune AB, Ware MD, Mazoyer S. The 185delAG mutation (c. 68_69delAG) in the BRCA1 gene triggers translation reinitiation at a downstream AUG codon. Hum Mutat. 2006; 27:1024-1029. [PubMed: 16941470]

21. Liu HX, Cartegni L, Zhang MQ, Krainer AR. A mechanism for exon skipping caused by nonsense or missense mutations in BRCA1 and other genes. Nat Genet. 2001; 27:55-58. [PubMed: 11137998]

22. Mazoyer S, Puget N, Perrin-Vidoz L, Lynch HT, Serova-Sinilnikova OM, Lenoir GM. A BRCA1 nonsense mutation causes exon skipping. Am J Hum Genet. 1998; 62:713-715. [PubMed: 9497265]

23. Perrin-Vidoz L, Sinilnikova OM, Stoppa-Lyonnet D, Lenoir GM, Mazoyer S. The nonsensemediated mRNA decay pathway triggers degradation of most BRCA1 mRNAs bearing premature termination codons. Hum Mol Genet. 2002; 11:2805-2814. [PubMed: 12393792]

24. Keiding, N. Delayed Entry. In: Armitage, P.; Colton, T., editors. Encyclopedia of Biostatistics. Hoboken, NJ: John Wiley; 2005.

25. Lakhani SR, van de Vijver MJ, Jacquemier J, et al. The Pathology of Familial Breast Cancer: Predictive Value of Immunohistochemical Markers Estrogen Receptor, Progesterone Receptor, HER-2, and p53 in Patients With Mutations in BRCA1 and BRCA2. Journal of Clinical Oncology. 2002; 20:2310-2318. [PubMed: 11981002]

26. Lakhani SR, Jacquemier J, Sloane JP, et al. Multifactorial Analysis of Differences Between Sporadic Breast Cancers and Cancers Involving BRCA1 and BRCA2 Mutations. Journal of the National Cancer Institute. 1998; 90:1138-1145. [PubMed: 9701363]

27. Moslehi R, Chu W, Karlan B, et al. BRCA1 and BRCA2 mutation analysis of 208 Ashkenazi Jewish women with ovarian cancer. Am J Hum Genet. 2000; 66:1259-1272. [PubMed: 10739756]

28. Singer G, Oldt R, Cohen Y, et al. Mutations in BRAF and KRAS Characterize the Development of Low-Grade Ovarian Serous Carcinoma. Journal of the National Cancer Institute. 2003; 95:484486. [PubMed: 12644542]

29. Singer G, Stöhr R, Cope L, et al. Patterns of p53 Mutations Separate Ovarian Serous Borderline Tumors and Low- and High-grade Carcinomas and Provide Support for a New Model of Ovarian Carcinogenesis: A Mutational Analysis With Immunohistochemical Correlation. The American Journal of Surgical Pathology. 2005:29. [PubMed: 15613854] 
30. Meindl A, Hellebrand H, Wiek C, et al. Germline mutations in breast and ovarian cancer pedigrees establish RAD51C as a human cancer susceptibility gene. Nat Genet. 2010; 42:410-414. [PubMed: 20400964]

31. Gourley C, Michie CO, Roxburgh P, et al. Increased incidence of visceral metastases in scottish patients with BRCA1/2-defective ovarian cancer: an extension of the ovarian BRCAness phenotype. J Clin Oncol. 2010; 28:2505-2511. [PubMed: 20406939]

32. Press JZ, De LA, Boyd N, et al. Ovarian carcinomas with genetic and epigenetic BRCA1 loss have distinct molecular abnormalities. BMC Cancer. 2008; 8:17. [PubMed: 18208621]

33. Hennessy BT, Timms KM, Carey MS, et al. Somatic mutations in BRCA1 and BRCA2 could expand the number of patients that benefit from poly (ADP ribose) polymerase inhibitors in ovarian cancer. J Clin Oncol. 2010; 28:3570-3576. [PubMed: 20606085]

34. Integrated genomic analyses of ovarian carcinoma. Nature. 2011; 474:609-615. [PubMed: 21720365]

35. Chiang JW, Karlan BY, Cass L, Baldwin RL. BRCA1 promoter methylation predicts adverse ovarian cancer prognosis. Gynecol Oncol. 2006; 101:403-410. [PubMed: 16360812]

36. Fong PC, Boss DS, Yap TA, et al. Inhibition of poly(ADP-ribose) polymerase in tumors from BRCA mutation carriers. N Engl J Med. 2009; 361:123-134. [PubMed: 19553641]

37. Fong PC, Yap TA, Boss DS, et al. Poly(ADP)-ribose polymerase inhibition: frequent durable responses in BRCA carrier ovarian cancer correlating with platinum-free interval. J Clin Oncol. 2010; 28:2512-2519. [PubMed: 20406929]

38. Thompson D, Easton D. Variation in BRCA1 cancer risks by mutation position. Cancer Epidemiol Biomarkers Prev. 2002; 11:329-336. [PubMed: 11927492]

39. Thompson D, Easton D. Variation in cancer risks, by mutation position, in BRCA2 mutation carriers. Am J Hum Genet. 2001; 68:410-419. [PubMed: 11170890]

40. Gayther SA, Mangion J, Russell P, et al. Variation of risks of breast and ovarian cancer associated with different germline mutations of the BRCA2 gene. Nat Genet. 1997; 15:103-105. [PubMed: 8988179]

41. Graham J, Olchowski A, Gilreath T. How Many Imputations are Really Needed? Some Practical Clarifications of Multiple Imputation Theory. Prevention Science. 2007; 8:206-213. [PubMed: 17549635]

42. Royston P. Multiple imputation of missing values. Stata Journal. 2004; 4:227-241.

43. van Buuren S, Boshuizen HC, Knook DL. Multiple imputation of missing blood pressure covariates in survival analysis. Statist Med. 1999; 18:681-694.

44. RUBIN DB. Inference and missing data. Biometrika. 1976; 63:581-592.

45. White IR, Royston P. Imputing missing covariate values for the Cox model. Statist Med. 2009; 28:1982-1998.

46. Moons KGM, Donders RART, Stijnen T, Harrell J. Using the outcome for imputation of missing predictor values was preferred. Journal of Clinical Epidemiology. 2006; 59:1092-1101. [PubMed: 16980150] 


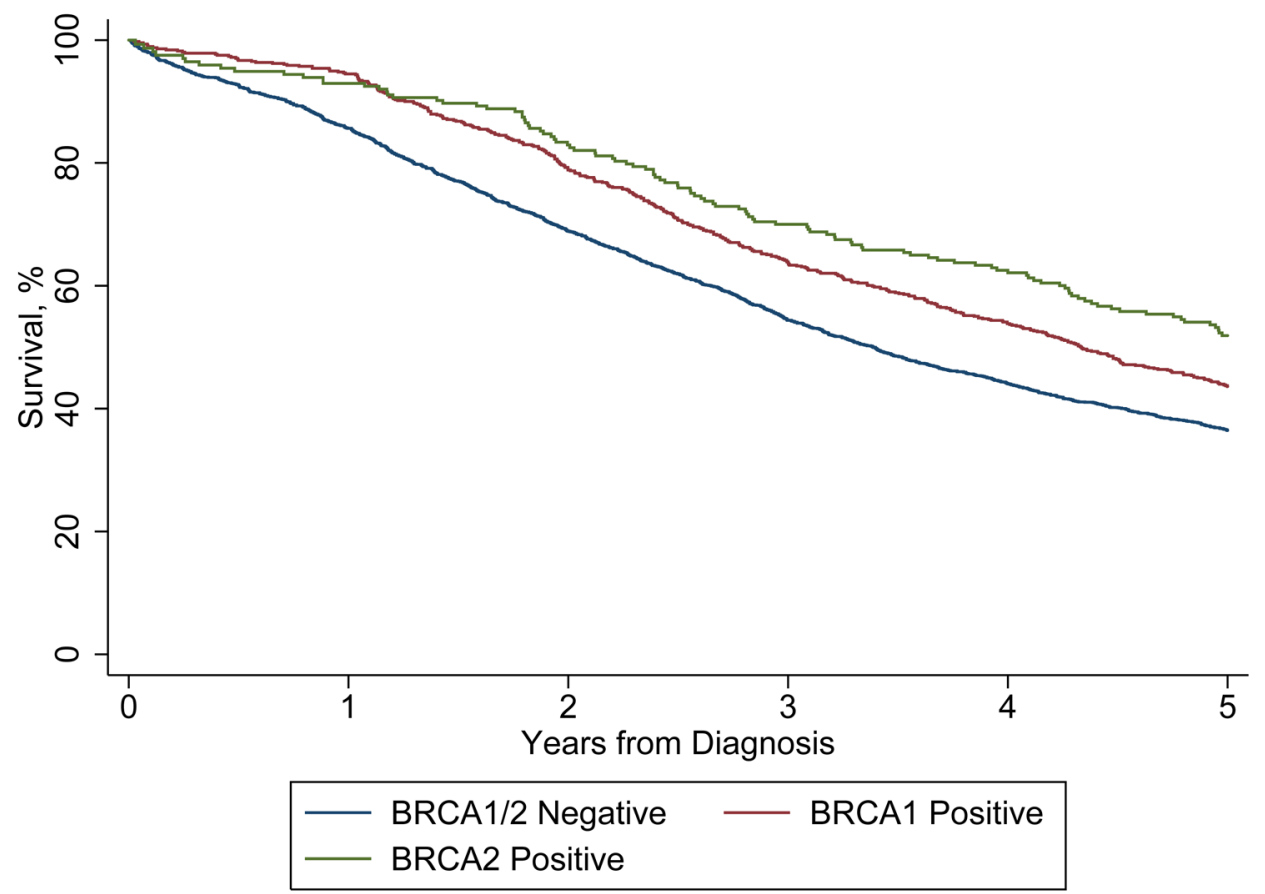

Figure 1. Kaplan Meier Estimates of Cumulative Survival According to BRCA1/2 status Caption: Kaplan Meier analysis was adjusted for year of diagnosis and study. 


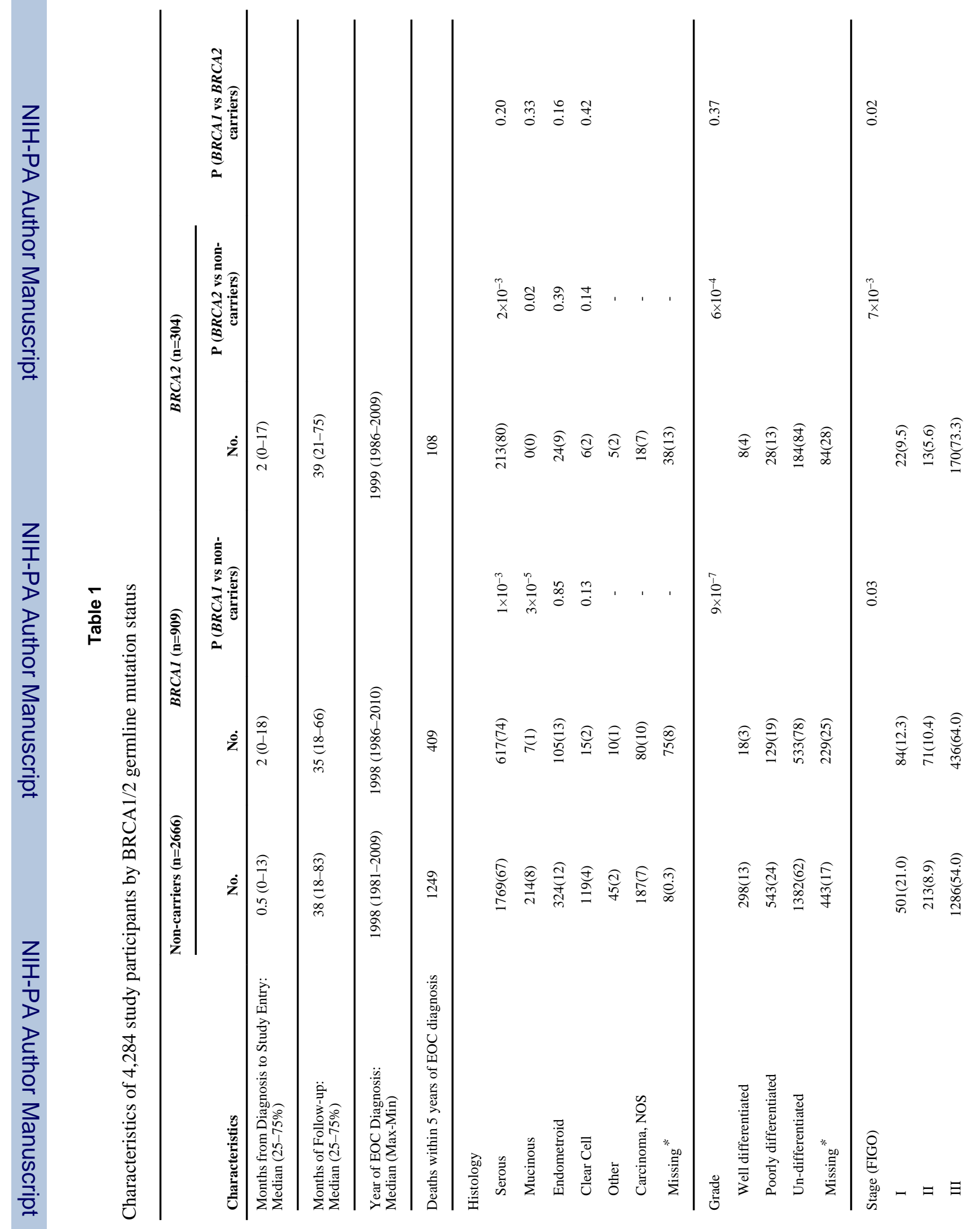




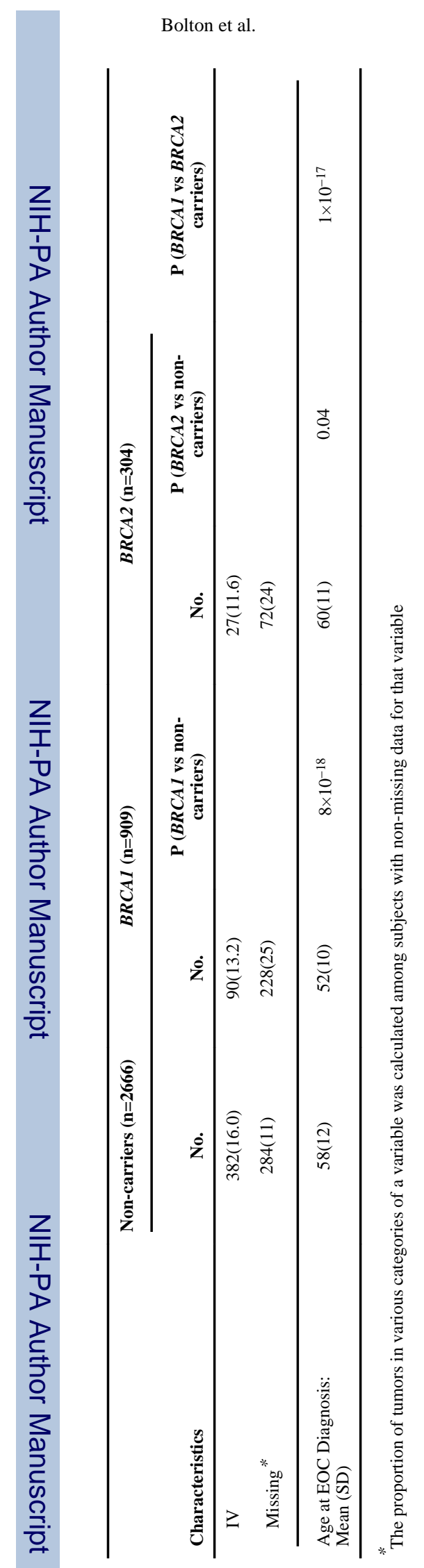

Page 15 


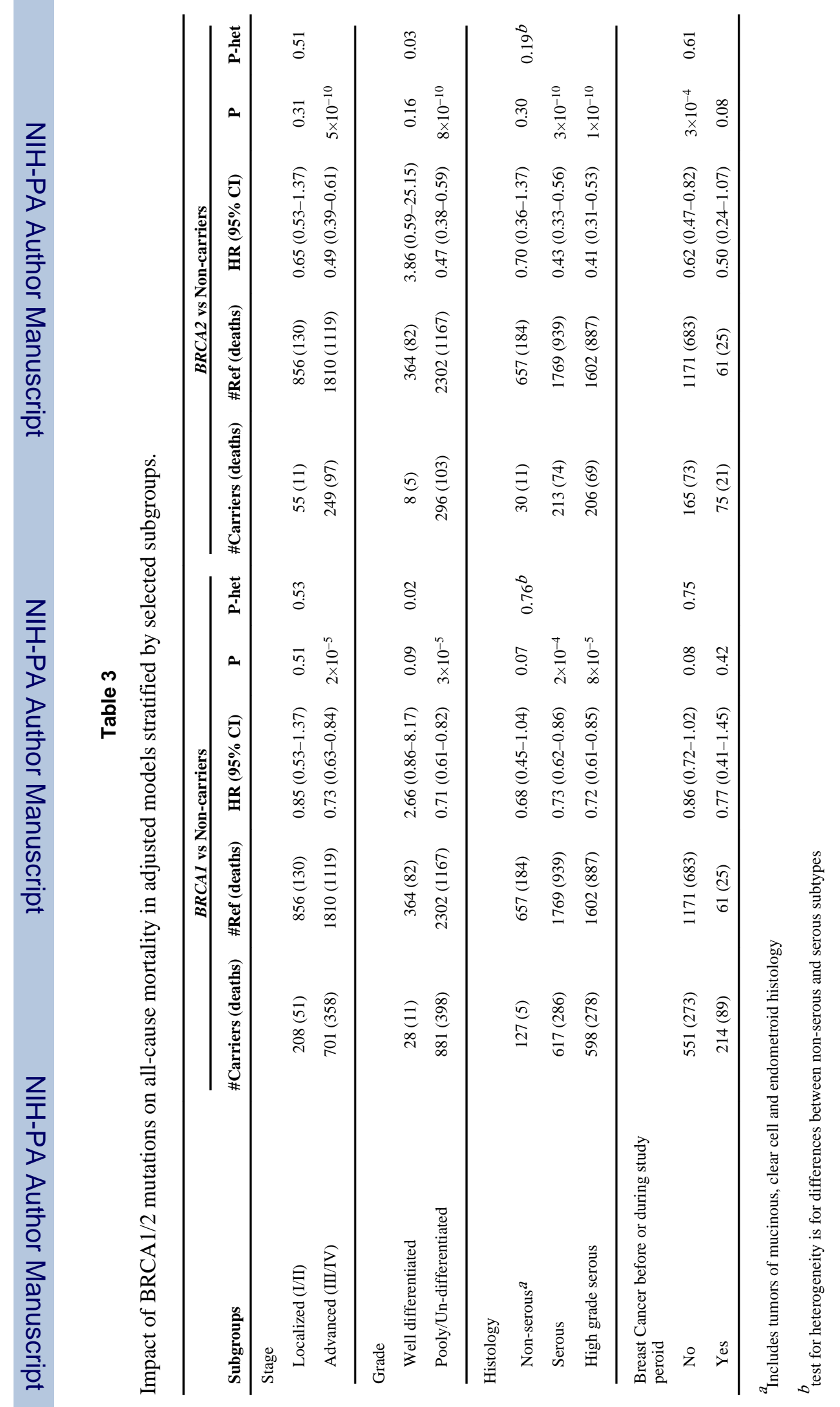




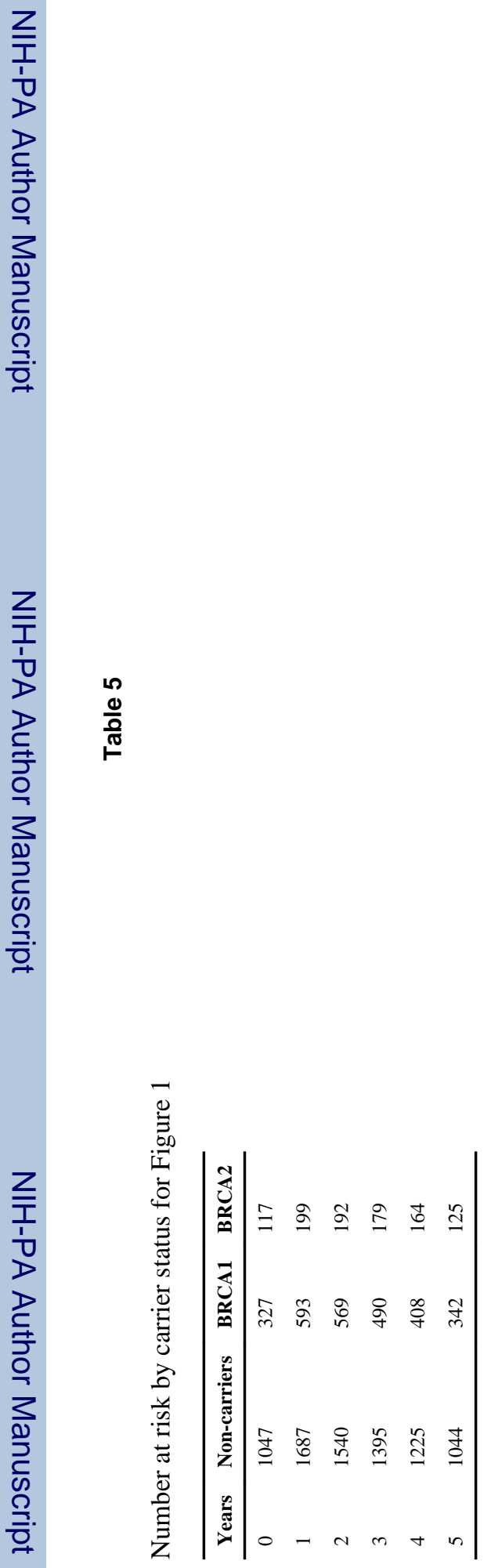

